\title{
Relativistic Mechanism of Chiral Magnetic Current in Weyl Semimetals with Tilted Dispersion
}

\author{
Zaur Z. Alisultanov \\ Amirkhanov Institute of Physics, Russian Academy of Sciences, \\ Dagestan Science Centre, Makhachkala, Russia. and \\ Dagestan State University, Makhachkala, Russia. ${ }^{*}$
}

\begin{abstract}
The chiral magnetic effect is a one of the exotic bulk transport properties of the Weyl semimetals. Because of the Nielsen-Ninomiya "no-go theorem", the total chiral magnetic current is absent in the equilibrium state. One of the mechanisms for generating this current is the chiral anomaly. This phenomenon is the anomalous nonconservation of chiral charge for massless relativistic particles. It can be realized by parallel magnetic and electric fields $(\sim \mathbf{E} \cdot \mathbf{B})$, and it leads to such new transport phenomenon as the negative longitudinal magnetoresistance. Using a simple theory (we consider both linearized and lattice model), we have shown, that in Weyl metals with tilted dispersion another mechanism of the chiral magnetic current is possible. It is not associated with the chiral anomaly. The new transport mechanism is based on the relativistic effect of electric field on Landau levels. This effect is that an electric field changes the distance between the Landau levels, and also changes the effective velocity along magnetic field. At presence of a tilt in the spectrum, this velocity renormalization is differ for different Weyl points. This leads to a non-zero resulting drift velocity. As a consequence, an electrical current arises along the magnetic field. The induced by this mechanism the electric current is proportional to the pseudoscalar product of the fields $(\mathbf{E} \vee \mathbf{B})$ and directed along the magnetic field, that differs it from the Hall current $(\sim \mathbf{E} \times \mathbf{B})$. At the same time, the conductivity corresponding to this transport mechanism does not depend on the scattering time like the Hall conductivity. Thus, we have proposed a new anomalous transport mechanism in the Weyl semimetal, which is not associated with the chiral anomaly.
\end{abstract}

\section{INTRODUCTION}

Materials with a non-trivial topology of the band structure are of great interest for both fundamental and practical application purposes (see, for example, [1 6]). The key property of quasi-particles in such materials is their chirality. The main consequence of a nontrivial topology and chirality is the topological stability (protection) of electronic states. Such stability is based on two conditions. First, the spectrum of carriers should be linear (Dirac fermions), since in this case chirality becomes a good quantum number. Indeed, the massless particles retain chirality: $\left[\mathcal{H}, \gamma^{5}\right]=0$, where $\mathcal{H}=\gamma_{0} \gamma_{\mu} p^{\mu}$ is the Hamiltonian of the massless particles, $\gamma^{5}=i \gamma_{0} \gamma_{1} \gamma_{2} \gamma_{3}$ is the chirality, $\gamma_{\mu}$ are Dirac matrices. The second condition consists in the spatial separation of states with opposite chiralities. This condition imposes certain restrictions on the dimensions of chiral electron states. In a 1D system, one can create zero-dimensional chiral states localized at opposite ends. For two dimensions, opposite chiralities can be separated to opposite edges of the plane, forming one-dimensional edge chiral states (2D topological insulator). In a 3D system, the opposite chiralities can be separated to opposite surfaces. The $2 \mathrm{D}$ chiral states (3D topological insulator) can be created this way. Continuing this scheme we can come to the conclusion that 3D chiral states occur at the edges of the four-dimensional system. Formally, such a situation has already been considered [7]. However, there is another scheme, which is

\footnotetext{
* zaur0102@gmail.com
}

more realistic and already experimentally implemented. It consists in the fact that opposite chiralities should be separated in some other space of parameters, for example, the momentum space. Such materials are called 3D Weyl semimetals (WSMs) 8-11. Without doping the chemical potential in such materials is localized at the Weyl point (WP). In this paper we consider the doped WSMs with the non-zero chemical potential, calculated from the WP.

The non-triviality of band structure of WSMs results in special chiral kinetics [12. These materials have a number of exotic transport properties, such as the anomalous Hall effect, the chiral magnetic effect and negative magnetoresistance. In this work, we are interested in the second of these effects. Because of the Nielsen-Ninomiya "no-go theorem", the total chiral magnetic current is absent in the equilibrium state. However, the non-zero chiral electronic transport in the WSM can be induced by the chiral anomaly [13 17]. This quantum phenomenon is the anomalous nonconservation of chiral charge for Weyl particles under the parallel magnetic and electric fields $\left(d\left(n_{R}-n_{L}\right) / d t \sim \mathbf{E} \cdot \mathbf{B}\right)$. The chiral anomaly leads to such new transport phenomenon as the negative longitudinal magnetoresistance. In this paper, using a simple theory we have shown, that in Weyl metals with tilted dispersion another mechanism of the chiral magnetic current is possible. It is not associated with the chiral anomaly. The new transport mechanism is based on the relativistic effect of electric field on Landau levels (LLs). This effect is that an electric field changes the distance between the LLs, and also changes the effective velocity along magnetic field. At presence of a tilt in 


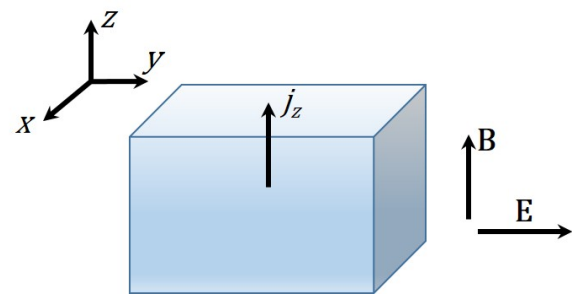

Figure 1. Schematic illustration of current along magnetic field. The electric field $\mathbf{E}$ is perpendicular to the electric current $\mathbf{j}$ and to the magnetic field $\mathbf{B}$.

the spectrum, this velocity renormalization is differ for different Weyl points. This leads to a non-zero resulting drift velocity. As a consequence, an electrical current arises along the magnetic field (see Fig. 1). The induced by this mechanism the electric current is proportional to the pseudoscalar product of the fields $(\mathbf{E} \vee \mathbf{B})$ and directed along the magnetic field, that differs it from the Hall current $(\sim \mathbf{E} \times \mathbf{B})$. Thus, we have proposed a new anomalous transport mechanism in the WSM, which is not associated with such exotic phenomena, like a chiral anomaly. The only exotic phenomenon in the proposed mechanism consists in the relativistic effect of the electric field on the LLs.

After introduction, the paper is organized as follows. In Sec. II we present a general properties of tilted Weyl Hamiltonian and LLs. We present conclusions about conditions which are imposed on the dispersion tilt. In Sec. III we consider the relativistic effect of electric field on the LLs and induced by such effect the new transport mechanism. We consider both linearized (III.A) and lattice model (III.C). In this chapter, we also give a general quasiclassical approach to the new effect (III.B). We conclude in Sec. IV with a some evaluations and discussion of the potential applications.

\section{HAMILTONIAN AND LANDAU LEVELS}

For the description of Weyl fermions with broken Lorentz-invariance (tilted dispersion) near the i-th WP we use a simple low-energy model [18]

$$
\mathcal{H}_{i}(\mathbf{p})=\boldsymbol{\omega}_{i} \cdot \mathbf{p}+k_{i} v_{i} \boldsymbol{\sigma} \mathbf{p}
$$

where $k_{i}=\operatorname{sign}\left\{\frac{1}{2 \pi} \oint \boldsymbol{b}_{i}(\mathbf{p}) \cdot d \mathbf{S}\right\}= \pm 1$ denotes the Chern number sign (chirality) of the WPs, $\boldsymbol{b}$ is the Berry curvature, $\boldsymbol{\omega}_{i}=\left(\omega_{i x}, \omega_{i y}, \omega_{i z}\right)$ spectrum tilt vector, $\boldsymbol{\sigma}$ is the Pauli matrices triplet, $\mathbf{p}$ is the quasiparticle momentum measured from WPs, $v_{i}$ is the Fermi velocity for i-th WP. Such a minimal model is quite sufficient to describe the basic properties of WSMs in a continuum approximation. The Hamiltonian (1) provides the spectrum

$$
\mathcal{E}_{p}^{i}=\boldsymbol{\omega}_{i} \cdot \mathbf{p} \pm v_{i} p
$$

where signs \pm refer to electrons and holes respectively. For velocity $\boldsymbol{v}=\partial \mathcal{E}_{p} / \partial \mathbf{p}$ we obtain

$$
\boldsymbol{v}_{i}=\boldsymbol{\omega}_{i}+k_{i} v_{i} \frac{\mathbf{p}}{p}
$$

The "no-go theorem" of Nielsen-Ninomiya theorem states that in lattice gauge theory the total Chern number of all WPs should be zero, i.e. $\sum_{i} k_{i} v_{i}=0\left(\right.$ term $\boldsymbol{\omega}_{i} \cdot \mathbf{p}$ does not change the Chern number). In particular, for two WPs this provides $v_{1,2}=v_{F}$.

Let's find out what values of parameter $\boldsymbol{\omega}_{i}$ are allowed to the Hamiltonian (1). For this we consider the electric current density in the equilibrium state

$$
\mathbf{j}_{e q}=\sum_{i} e \int f_{0}^{i} \boldsymbol{v}_{i} d^{3} \mathbf{p}
$$

where $f_{0}^{i}=\left(e^{\beta\left(\mathcal{E}_{p}^{i}-\mu\right)}+1\right)^{-1}$ is the Fermi-Dirac carrier distribution function for $i$-th WP, $\mu$ is the chemical potential. The total chiral current should be zero in equilibrium: $\mathbf{j}_{e q}=0$. This requirement leads to the odd the integrand

$$
\sum_{i} f_{0}^{i} \boldsymbol{v}_{i}(\mathbf{p})=-\sum_{i} f_{0}^{i} \boldsymbol{v}_{i}(-\mathbf{p})
$$

which can be transformed to

$$
\sum_{i} \frac{\boldsymbol{\omega}_{i}\left(x \cosh \left(\boldsymbol{\omega}_{i} \cdot \mathbf{p}\right)+1\right)-v_{F} \frac{\mathbf{p}}{p} x \sinh \left(\boldsymbol{\omega}_{i} \cdot \mathbf{p}\right)}{\left(x e^{\beta \boldsymbol{\omega}_{i} \cdot \mathbf{p}}+1\right)\left(x e^{-\beta \boldsymbol{\omega}_{i} \cdot \mathbf{p}}+1\right)}=0
$$

where we introduced $x=e^{\beta\left(v_{F} p-\mu\right)}$. This condition can be satisfied in a variety of ways depending on the number of WPs the system contains. The number of WPs depends on the type of broken symmetry. If the $\mathcal{T}$ symmetry (time reversal symmetry) is broken, the minimum number of WPs equals two. If the $\mathcal{P}$ symmetry (space inversion symmetry) is broken, the minimum number of points equals four. In the case with two WPs we obtain: $\boldsymbol{\omega}_{1}=-\boldsymbol{\omega}_{2}$. In the general case, for (6) the following condition is necessary

$$
\sum_{i} \omega_{i}=0
$$

However, it is not sufficient, because not all combinations satisfying (7) satisfy condition (6). In the general case, the tilt parameters are contained in the system in pairs: $\pm \boldsymbol{\omega}_{i}$, i.e. each tilt parameter has a mirrored partner. We note that conditions (6) does not contain the WP chirality. In case the number of WPs is more than two, generally speaking, there are many various options to satisfy condition 6). For example, if the number of WPs equals four, then conditions (7) can be satisfied with the following configurations: $\boldsymbol{\omega}_{1}=\boldsymbol{\omega}_{2}=\boldsymbol{\omega}, \boldsymbol{\omega}_{3}=\boldsymbol{\omega}_{4}=-\boldsymbol{\omega}$ or 
$\boldsymbol{\omega}_{1}=-\boldsymbol{\omega}_{2}, \boldsymbol{\omega}_{3}=-\boldsymbol{\omega}_{4}$. etc. Each case presents its own peculiarities in relation to the effect that is considered here. However, the essence this effect remains the same for all cases. Therefore, below we will consider only the case of two WPs. It is easy to generalize our analysis to the case with $2 N(N \in \mathbb{Z})$ WPs.

In magnetic field we have $\mathbf{p} \rightarrow \mathbf{p}+\frac{e}{c} \mathbf{A}$. Let the magnetic field be directed along the $\mathrm{Z}^{c}$ axis, i.e. $\mathbf{B}=(0,0, B)$. We use the simplest Landau gauge: $\mathbf{A}=(-B y, 0,0)$. Applying double Lorentz-boost to the Hamiltonian 1 : $\mathcal{H}_{i} \rightarrow e^{\frac{\sigma_{y} \lambda_{2}^{i}}{2}} e^{\frac{\sigma_{x} \lambda_{1}^{i}}{2}} \mathcal{H}_{i} e^{\frac{\sigma_{x} \lambda_{1}^{i}}{2}} e^{\frac{\sigma_{y} \lambda_{2}^{i}}{2}}$, where $\tanh \lambda_{1}^{i}=\omega_{i x} / v_{F}$, $\tanh \lambda_{2}^{i}=\omega_{i y} / v_{F}$, we obtain the following expression for Landau spectrum [19 21]

$$
\mathcal{E}_{n}^{i}=\operatorname{sign}(n) v_{F} \sqrt{2 \gamma_{i}^{3} l_{H}^{-2} \hbar^{2} n+\gamma_{i}^{2} p_{z}^{2}}+\omega_{i z} p_{z}, \quad n \neq 0
$$

$$
\mathcal{E}_{0}^{i}=\left(k_{i} \gamma_{i} v_{F}+\omega_{i z}\right) p_{z} .
$$

where $\gamma_{i}=\sqrt{1-\frac{\omega_{i x}^{2}+\omega_{i y}^{2}}{v_{F}^{2}}}$. At presence of a magnetic field, the electric current along the $Z$ axis still does not appear. Indeed, electric current density $j_{z}$ in this case is determined as follows:

$$
j_{z}=\sum_{n, i} e \int_{-\infty}^{\infty} f_{0}^{i} v_{i z} d p_{z}
$$

It becomes clear that the total contribution of LLs with $v_{i z}=\partial E_{n} / \partial p_{z}$ equals zero due to the fact that integrand $\sum_{i} f_{0}^{i} v_{i z}$ is odd as a result of condition (6). Certainly, the magnetic field induces current near each WP as a result of the chiral magnetic effect: $\mathbf{j}_{C M E}=k_{i} \mathbf{B} \int\left(\frac{\mathbf{p}}{p} \cdot \boldsymbol{b}\right) f \frac{d^{3} \mathbf{p}}{(2 \pi)^{3}}$. However, the total electric current over all WPs will equal zero. This is a consequence of the Nielsen-Ninomiya "nogo theorem".

Below, for simplicity we suppose that $\omega_{i z}=0$.

\section{TRANSPORT INDUCED BY RELATIVISTIC EFFECT OF ELECTRIC FIELD}

\section{A. General consideration}

Let us now consider the effect of a perpendicular electric field on the LLs. Let the electric field is directed along the $Y$ axis: $\mathbf{E}=(0, E, 0)$, and the magnetic field is directed along the $\mathrm{Z}$ axis: $\mathbf{B}=(0,0, B)$. Then

$$
\tilde{\mathcal{H}}_{i}(\mathbf{p})=k_{i} v_{F} \boldsymbol{\sigma}\left(\mathbf{p}-\frac{e}{c} \mathbf{A}\right)+\boldsymbol{\omega}_{i} \cdot\left(\mathbf{p}-\frac{e}{c} \mathbf{A}\right)+e E y
$$

Applying double Lorentz-boost $\tilde{\mathcal{H}}_{i} \rightarrow$ $e^{\frac{\sigma_{x} \tilde{\lambda}_{1}^{i}}{2}} e^{\frac{\sigma_{y} \tilde{\lambda}_{2}^{i}}{2}} \tilde{\mathcal{H}}_{i} e^{\frac{\sigma_{x} \tilde{\lambda}_{1}^{i}}{2}} e^{\frac{\sigma_{y} \tilde{\lambda}_{2}^{i}}{2}}$ with $\tanh \tilde{\lambda}_{1}^{i}=\left(v_{0}-\omega_{i x}\right) / v_{F}$, $\tanh \tilde{\lambda}_{2}^{i}=\omega_{i y} / v_{F}$, we obtain [22 24]

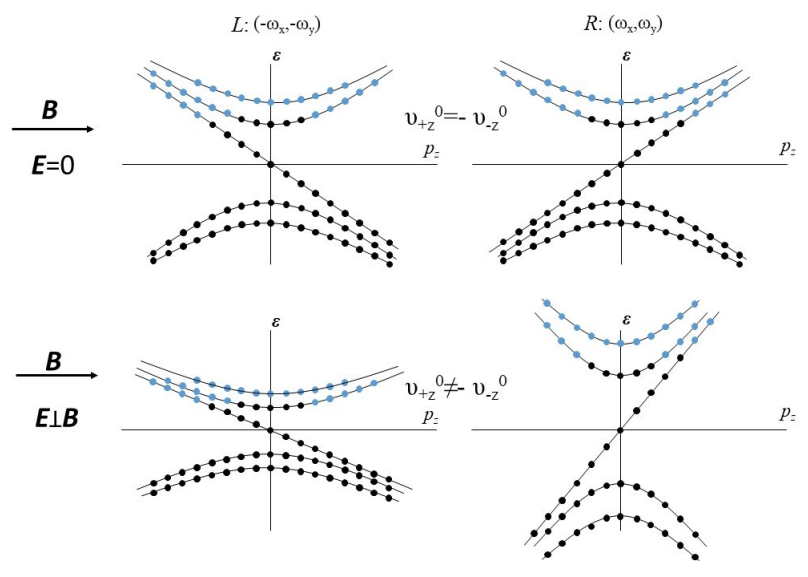

Figure 2. LLs (8), (9) (top part) and (11), 12 (bottom part) for pair of WPs with opposite chiralities. The tilt parameters are given as $\boldsymbol{\omega}_{i}=\left(k_{i} \omega_{x}, k_{i} \omega_{y}, 0\right)$. The perpendicular electric field affects the LLs velocity in different ways for different WPs.

$$
\tilde{\mathcal{E}}_{n}^{i}=\operatorname{sign}(n) v_{F} \sqrt{2 \tilde{\gamma}_{i}^{3} l_{H}^{-2} \hbar^{2} n+\tilde{\gamma}_{i}^{2} p_{z}^{2}}+v_{0} p_{x}, \quad n \neq 0
$$

$$
\tilde{\mathcal{E}}_{0}^{i}=k_{i} \tilde{\gamma}_{i} v_{F} p_{z}+v_{0} p_{x} .
$$

where $\tilde{\gamma}_{i}=\sqrt{1-\frac{\left(v_{0}-\omega_{i x}\right)^{2}+\omega_{i y}^{2}}{v_{F}^{2}}}, \boldsymbol{v}_{0}=c \frac{[\mathbf{E} \times \mathbf{B}]}{B^{2}}$. As can be seen, the electric field affects not only the cyclotron frequency, but also the velocity along the Z-axis. This is a purely relativistic effect. For the nonrelativistic spectrum, all the velocity components are independent: $v_{s}=\frac{p_{s}}{m}, s=x, y, z$. In the case of relativistic spectrum $\epsilon=c \sqrt{p^{2}+m^{2} c^{2}}$ each velocity component depends of all momentum components: $v_{s}=\frac{c p_{s}}{\sqrt{p^{2}+m^{2} c^{2}}}$. Therefore, the applied electric field affects all velocity components, although the momentum change only occur in the direction along which the field is applied. LLs in the presence and absence of an electric field are presented in Fig. 2. The tilt parameters in the figure are given as $\boldsymbol{\omega}_{i}=\left(k_{i} \omega_{x}, k_{i} \omega_{y}, 0\right)$. As can be seen from the figure, the electric field affects the LLs velocity in different ways for different WPs since $\boldsymbol{\omega}_{1}=-\boldsymbol{\omega}_{2}$.

The main idea of this paper can be understood already from formulas 11,12 . The total carrier velocity contribution from non-zero LLs still equals zero because this velocity for the given WP is an odd function of momentum $p_{z}$. At the same time the total velocity of zero levels from all WPs $\left(v_{z}^{0}=\sum_{i} k_{i} \tilde{\gamma}_{i} v_{F}\right)$ now does not vanish since $\tilde{\gamma}_{1} \neq \tilde{\gamma}_{2}$. This is the result of the fact that the electric field has different impact on tilted spectrum near different WPs. In the case with two WPs, when $\boldsymbol{\omega}_{i}=\left(k_{i} \omega_{x}, k_{i} \omega_{y}, 0\right)$, we obtain

$$
v_{1 z}^{0}+v_{2 z}^{0}=\left[\tilde{\gamma}_{+}-\tilde{\gamma}_{-}\right] v_{F},
$$


where $\tilde{\gamma}_{ \pm}=\sqrt{1-\frac{\left(v_{0} \mp \omega_{x}\right)^{2}+\omega_{y}^{2}}{v_{F}^{2}}}$. Next, we are interested in the response of system to a weak electric field. Then, in linear response limit for the last expression we have the following:

$$
v_{1 z}^{0}+v_{2 z}^{0} \approx \frac{2 \omega_{x}}{\gamma_{0}} \frac{v_{0}}{v_{F}}
$$

where $\gamma_{0}=\sqrt{1-\frac{\omega_{x}^{2}+\omega_{y}^{2}}{v_{F}^{2}}}$. This expression provides the main contribution in linear approximation. Let us consider this issue in more detail. First of all, the smallness of the electrical field allows us to neglect the $v_{0} p_{x}$ term in the spectrum. Therefore, for the distribution function in linear approximation we obtain

$$
f^{i}=\frac{1}{e^{\beta\left(\tilde{\mathcal{E}}_{0}^{i}-\mu\right)}+1} \approx f_{0}^{i}+\delta f^{i} \frac{\omega_{x}}{\gamma_{0}} \frac{v_{0}}{v_{F}^{2}}
$$

where $\delta f^{i}=\frac{\beta v_{F} p_{z}}{4 \cosh ^{2}\left(\frac{1}{2} \beta\left(\mathcal{E}_{0}^{i}-\mu\right)\right)}$. Then

$$
f^{1} v_{1 z}^{0}+f^{2} v_{2 z}^{0} \approx\left(f_{0}^{1}+f_{0}^{2}+\left(\delta f^{1}-\delta f^{2}\right) \gamma_{0}\right) \frac{\omega_{x}}{\gamma_{0}} \frac{v_{0}}{v_{F}}
$$

In the right part of the last expression we left only linear field terms and neglected the terms that disappear after integration. Here we should note that strictly speaking chirality conservation and absence of scattering in WSMs are imperfect. Indeed, we can talk about chirality conservation only when we use the low-energy approximation for Hamiltonian. Strictly speaking, even the chiral energy level is characterized by relaxation time $\tau_{c}$. This time can be estimated with the help of the following expression: $\frac{1}{\tau_{c}} \sim \frac{\epsilon_{F}^{2}}{W^{2} \tau}$, where $W$ is the parameter that characterizes the band width. However, in this work we neglect collisions for chiral levels assuming that the Fermi level is sufficiently close to the WP.

So, the contribution of zero LLs to electric current density in linear approximation is as follows:

$$
j_{z}^{0}=\left(\sigma_{z y}^{0}+\sigma_{z y}^{\delta}\right) E_{y}
$$

where

$$
\begin{gathered}
\sigma_{z y}^{0}=\frac{e \omega_{x}}{\gamma_{0}} \frac{c}{v_{F} B} n_{0}, \\
\sigma_{z y}^{\delta}=\frac{e \omega_{x}}{\gamma_{0}} \frac{c}{v_{F} B} n_{\delta},
\end{gathered}
$$

where $n_{0}=\int_{-\infty}^{\infty}\left(f_{0}^{1}+f_{0}^{2}\right) d p_{x} d p_{z}$ is the concentration of carriers on the zero LLs and $n_{\delta}=$ $\gamma_{0} \int_{-\infty}^{\infty}\left(\delta f^{1}-\delta f^{2}\right) d p_{x} d p_{z}$. After integration we obtain:

$$
\sigma_{z y}=\sigma_{z y}^{0}+\sigma_{z y}^{\delta}=\frac{e^{2}}{(2 \pi \hbar)^{2}} \frac{\omega_{x}}{\gamma_{0}^{2}} \frac{2 \mu}{v_{F}^{2}}\left[\frac{\ln \left(1+e^{\beta \mu}\right)}{\beta \mu}+1\right],
$$

where we took into account that $\int d p_{x}=\frac{e B}{c}$. We should note that this conductivity is finite in the collisionless regime considered here. Moreover, even if the collisions are taken into account, value $\sigma_{z y}^{0}$ does not depend on the scattering time. This property makes the considered effect similar to the Hall effect. We can introduce a constant similar to the Hall constant: $R_{\omega}=$ $\frac{E_{y}}{j_{z}^{0} H}=\frac{v_{F} \gamma_{0}}{2 e\left(n_{0}+n_{\delta}\right) \omega_{x} c}$. Such independence on the scattering sharply distinguishes this transport mechanism from the chiral anomaly. The presence of scattering is extremely important for observing a chiral anomaly in a condensed matter. Indeed, due to the crystal potential periodicity, the electron dispersion law is bounded, that will affect the continuous transfer of carriers from one Weyl point to another. The presence of a relaxation mechanism cuts off the effect of a periodic crystal potential. The transport mechanism considered by us does not need an introduction of the any relaxation mechanism. This is due to the fact that in this mechanism the transport is determined by the pseudoscalar product of the fields and the momentum $p_{z}$ is the integral of motion. At low temperatures $\beta \mu \gg 1$ we can evaluate this conductivity:

$$
\sigma_{z y} \approx \frac{e^{2}}{(\pi \hbar)^{2}} \frac{\omega_{x}}{\gamma_{0}^{2}} \frac{\epsilon_{F}}{v_{F}^{2}}
$$

Now let's calculate the contribution to current from non-zero LLs. These levels are not have chiral, so the scattering time for them is finite. Using the $\tau$ approximation approach which is a simplest model for collision integral, we obtain

$$
\begin{gathered}
j_{z}^{n \neq 0} \propto \sum_{n, i} \int_{-\infty}^{\infty} \delta f^{i} v_{i z}^{n} \frac{d p_{x} d p_{z}}{(2 \pi \hbar)^{2}}= \\
=\frac{E_{y}}{(2 \pi \hbar)^{2}} \sum_{n, i} \int d p_{x} \tau(\varepsilon) \frac{\partial}{\partial p_{y}} \int f_{0}^{i} v_{i z}^{n} d p_{z}=0
\end{gathered}
$$

The reason this contribution is absent is that the integrand expression remains an odd function of $p_{z}$ even in the presence of an electric field. It is important that for s-scattering even the full distribution function remains an even function of $p_{z}$ in the presence of the $E_{y}$ field. So, in this case formula $(18)$ is valid not only in linear approximation. Thus, the electric current appears only due to zero chiral LLs. In order to completely distract ourselves from the contribution of non-zero LLs, we consider here the case of weakly-doped WSMs, when all electrons are located at the zero level.

Finally, we note that term $v_{0} p_{x}$ omitted above does not affect our calculations. If we reconstruct this term in the spectrum, then in a linear approximation, an additional term proportional to $v_{0} p_{x}$ appears in formula (14). However, after integrating over $p_{x}$ this term vanishes: $\int_{-e B L_{y} / 2 c}^{e B L_{y} / 2 c} p_{x} d p_{x}=0$. 


\section{B. Quasiclassical chiral kinetic approach}

Now we investigate our problem in a general form. To do this, we consider the quasiclassical kinetic theory for a system in which different WPs are characterized by the usual Weyl Hamiltonians, but with different Fermi velocities. Hamiltonian near the i-th $\mathrm{WP}(i=1,2)$ we write in the form

$$
\mathcal{H}_{i}(\mathbf{p})=k_{i} v_{i} \boldsymbol{\sigma} \mathbf{p}
$$

where $v_{i}$ is the Fermi velocity in the i-th Weyl point. Using this Hamiltonian we write the expression for the classic action

$$
\mathcal{S}_{i}=\int_{t_{a}}^{t_{b}} d t\left\{\mathbf{p} \frac{d \mathbf{x}}{d t}-k_{i} v_{i} \boldsymbol{\sigma} \mathbf{p}\right\}
$$

Using the standard method of diagonalization of the action (see, for example, [12]) and adding an electromagnetic field, we get

$$
\mathcal{S}_{i}=\int_{t_{a}}^{t_{b}} d t\left\{\left(\mathbf{p}+\frac{e}{c} \mathbf{A}\right) \frac{d \mathbf{x}}{d t}-e \Phi-v_{i} p-k_{i} \hbar \boldsymbol{a}_{\mathbf{p}} \cdot \frac{d \mathbf{p}}{d t}\right\}
$$

where $\Phi, \mathbf{A}$ are the scalar and vector potentials, $\mathbf{a}_{p}=$ $i V_{p}^{\dagger} \boldsymbol{\nabla}_{\mathbf{p}} V_{p}$ is Berry connection and

$$
V_{p}=\frac{1}{\sqrt{2 p\left(p-p_{z}\right)}}\left(\begin{array}{cc}
p_{x}-i p_{y} & -p+p_{z} \\
p-p_{z} & p_{x}+i p_{y}
\end{array}\right)
$$

In equilibrium state, as we saw above, $v_{i}=v_{F}$. Now we use this parameter in general form, assuming that the Fermi velocities of different points differ in magnitude for some reason (of course, this state is not equilibrium). The Eq. 20 gives the following equations of motion (we use the Hamilton formalism)

$$
\begin{gathered}
\frac{d \mathbf{p}}{d t}=\frac{e}{c}\left[\frac{d \mathbf{x}}{d t} \times \mathbf{B}\right]+e \mathbf{E} \\
\frac{d \mathbf{x}}{d t}=v_{i} \frac{\mathbf{p}}{p}+k_{i} \hbar\left[\frac{d \mathbf{p}}{d t} \times \boldsymbol{b}\right]
\end{gathered}
$$

where $\boldsymbol{b}=\nabla_{\mathbf{p}} \times \mathbf{a}_{p}=\mathbf{p} / 2 p^{3}$ is the Berry curvature. Using these equations you can get the quasiclassical kinetic equation and current density

$$
\begin{aligned}
\mathbf{j}_{i}=e v_{i} \int_{\mathbf{p}} \frac{\mathbf{p}}{p} f+k_{i} \hbar e^{2}\left[\mathbf{E} \times \int_{\mathbf{p}} f \boldsymbol{b}\right]+ \\
+k_{i} v_{i} \frac{e^{2}}{c} \hbar \mathbf{B} \int_{\mathbf{p}} f\left(\frac{\mathbf{p}}{p} \cdot \boldsymbol{b}\right)
\end{aligned}
$$

The first term of right part describes the usual non-chiral current. The contribution from nonzero LLs, which we calculated in Eq. 18 refers to this term. The second term describes the anomalous quantum Hall effect. Note that this effect is determined by the Berry curvature and the distance between the WPs. The relativistic effect of the electric field, discussed here, has no affect this term. The third term describes the chiral-magnetic effect

$$
\mathbf{j}_{C M E}^{i}=k_{i} v_{i} \frac{e^{2}}{c} \hbar \mathbf{B} \int_{\mathbf{p}} f\left(\frac{\mathbf{p}}{p} \cdot \boldsymbol{b}\right)
$$

The chiral magnetic current in this form depends both on the sign $\left(k_{i}\right)$ and on the magnitude $\left(v_{i}\right)$ of the velocity. This expression can be reduced to the more simple and clear form at zero temperature. Taking into account that

$$
\int_{\mathbf{p}} f\left(\frac{\mathbf{p}}{p} \cdot \boldsymbol{b}\right)=v_{i}^{2} \int_{0}^{\mu_{i}} \frac{\rho(\epsilon) d \epsilon}{2 \epsilon^{2}}=\frac{\mu_{i}}{4 \pi^{2} \hbar^{3} v_{i}},
$$

we get that the chiral current is determined by the chemical potentials of WPs

$$
j_{C M E}=\frac{e^{2}}{4 \pi^{2} \hbar^{2} c} \sum_{i} k_{i} \mu_{i} B
$$

This shows that the total chiral current is nonzero only if the chemical potentials of different WPs are different: $\mu_{1} \neq \mu_{2}$. This can be achieved, for example, by chiral anomaly. In this case

$$
\mu_{i}=\mu_{0}+k_{i} v_{i} e E \tau
$$

where $\tau$ is the transport relaxation time. Then

$$
j_{C A E}=\frac{e^{3}}{4 \pi^{2} \hbar^{2} c}\left(v_{1}+v_{2}\right) \tau E B
$$

As we can see, this current depends on the Fermi velocity at both WPs. If $v_{1}=v_{2}=v_{F}$ we get the well-known formula for anomaly-induced current.

Let us now apply Eq.(24) to describe the relativistic mechanism of chiral transport. As shown above, the transverse electric field changes the velocity of the zero LL. I.e. the electric field is an external perturbation that leads to inequality of velocities $v_{1} \neq v_{2}$. It is easy to show that this leads to the inequality of the chemical potentials at different WPs, i.e. $\mu_{1} \neq \mu_{2}$. In other words, the equality of chemical potentials in different Weyl cones can be violated not only by the imbalance of the chiral charge (chiral anomaly), but also by changing the angles of these cones (Fermi velocities). Indeed, in the absence of an electric field we have $v_{1}=v_{2}=v_{F}$. Then the carrier concentration near each of the Weyl points is defined as follow 


$$
n=\int_{0}^{p_{0}} \frac{4 \pi p^{2} d p}{(2 \pi \hbar)^{3}}=\frac{1}{2 \pi^{2} \hbar^{3} v_{F}^{3}} \frac{\mu_{0}^{3}}{3}
$$

or

$$
\mu_{0}=v_{F} \hbar\left(6 \pi^{2} n\right)^{1 / 3} .
$$

The electric field leads to a violation of the equality of velocities, i.e. $v_{1} \neq v_{2}$. Consequently,

$$
\begin{aligned}
& \mu_{1}=v_{1} \hbar\left(6 \pi^{2} n\right)^{1 / 3}=\frac{\mu_{0}}{v_{F}} v_{1} \\
& \mu_{2}=v_{2} \hbar\left(6 \pi^{2} n\right)^{1 / 3}=\frac{\mu_{0}}{v_{F}} v_{2}
\end{aligned}
$$

Then

$$
j_{C M E}=\frac{e^{2}}{4 \pi^{2} \hbar^{2}} \frac{\mu_{0}}{v_{F}} B\left(v_{1}-v_{2}\right)
$$

This is the semiclassical explanation of the effect discussed above. Using this formula and taking into account (13) we can easily obtain the formula (17). Of course, it should be noted that the current (26) disappears in the absence of doping, while the anomaly-induced current does not depend on the doping.

Once again we note that an important difference between relativistic mechanism and chiral anomaly is that first of them does not need an introduction of the relaxation time, while it is vital for the second.

Note that in a more rigorous model we need to take into account that the two WPs are connected to each other. Indeed, the WPs are different points of a single continuous Brillouin zone. In order to take this into account, we need to consider the lattice model. The calculations for the lattice model in the simplest form are given in the next chapter.

\section{Calculations for lattice model}

Finally, we briefly generalize the above results for the lattice model. We use the simplest model of the Weyl semimetal with a broken $\mathcal{T}$ symmetry, namely, the Burkov-Balent model. This model based on a magnetically doped TI-NI (TI is topological insulator and NI is normal insulator) multilayer heterostructure. This Hamiltonian has the form [25]

$$
\mathcal{H}_{B B}=v_{F} \tau^{z}[\hat{z} \times \boldsymbol{\sigma}] \cdot \mathbf{p}_{\perp}+m \sigma_{z}+\hat{\Delta}\left(k_{z}\right)
$$

where $\hat{\Delta}\left(k_{z}\right)=\Delta_{S} \tau^{x}+\frac{1}{2}\left(\Delta_{D} \tau^{+} e^{i k_{z} d}+\right.$ h.c. $), \mathbf{p}_{\perp}=$ $\left(p_{x}, p_{y}\right)$ is the momentum in the $2 \mathrm{D}$ surface Brillouin zone, $\boldsymbol{\tau}=\left(\tau_{x}, \tau_{y}, \tau_{z}\right)$ are Pauli matrices, acting on the which surface pseudospin degree of freedom, $m$ describes exchange spin splitting of the surface states, $\Delta_{S}$ and
$\Delta_{D}$ describe tunneling between top and bottom surfaces within the same TI layer $\left(\Delta_{S}\right)$, and between top and bottom surfaces of neighboring TI layers $\left(\Delta_{D}\right)$. Here we consider the case when $\Delta_{D}$ and $\Delta_{S}$ have opposite signs. Now we generalize this model by adding a tilt term in simplest form $\boldsymbol{\omega}_{i}=\left(\omega_{x}\left(k_{z}\right), 0,0\right)$. A simple analysis shows that the parameter $\omega_{x}$ in the lattice model (27) must be a periodic function of $k_{z}$. This function can be defined as $\omega_{x}\left(k_{z}\right)=\omega_{x} \sin \left(\frac{\pi k_{z}}{2 k_{0}}\right)$. At Weyl points $\pm k_{0}$ we get $\omega_{x}\left(k_{z}\right)= \pm \omega_{x}$, and in between these points the function $\omega_{x}\left(k_{z}\right)$ changes smoothly. Then for the tilted Hamiltonian we can write

$$
\begin{array}{r}
\tilde{\mathcal{H}}_{B B}=v_{F} \tau^{z}[\hat{z} \times \boldsymbol{\sigma}] \cdot\left(\mathbf{p}+\frac{e}{c} \mathbf{A}\right)+m \sigma_{z}+ \\
+\omega_{x} \sin \left(\frac{\pi k_{z}}{2 k_{0}}\right)\left(p_{x}+\frac{e}{c} A_{x}\right)+\hat{\Delta}\left(k_{z}\right)+e E y
\end{array}
$$

where we have included electric and magnetic fields in the same geometry as above in text, $\mathbf{A}=(-B y, 0,0)$. Applying now a non-uniform Lorentz-boost

$$
\tilde{\mathcal{H}}_{B B} \rightarrow e^{\tau^{z} \sigma_{y} \frac{\tilde{\lambda}_{1}}{2}} \tilde{\mathcal{H}}_{B B} e^{\tau^{z} \sigma_{y} \frac{\tilde{\lambda}_{1}}{2}}
$$

with $\tanh \tilde{\lambda}_{1}=\left(\omega_{x} \sin \left(\frac{\pi k_{z}}{2 k_{0}}\right)-v_{0}\right) / v_{F}=\tilde{\beta}$, we reduce the eigenvalues problem of Hamiltonian (28) to eigenvalues problem of such Hamiltonian without electric field and with effective magnetic field $B \sqrt{1-\tilde{\beta}^{2}}$. The eigenvalue problem for the Hamiltonian (27) in a quantizing magnetic field was solved in the paper [26]. After the diagonalization of the operator $\hat{\Delta}\left(k_{z}\right)$, the Hamiltonian can be represented in block form. This leads to four branches of the spectrum. We are interested only in those branches that contain WPs. Considering only such solutions, for the Hamiltonian spectrum 28 we finally get

$$
\tilde{\mathcal{E}}_{n}= \pm v_{F} \sqrt{2 \tilde{\gamma}_{L}^{3} l_{H}^{-2} \hbar^{2} n+\tilde{\gamma}_{L}^{2}\left(m-\Delta\left(p_{z}\right)\right)^{2}}+v_{0} p_{x}
$$

$$
\tilde{\mathcal{E}}_{0}=\tilde{\gamma}_{L}\left(m-\Delta\left(p_{z}\right)\right)+v_{0} p_{x} .
$$

where $\tilde{\gamma}_{L}=\sqrt{1-\frac{\left(\omega_{x} \sin \left(\frac{\pi k_{z}}{2 k_{0}}\right)-v_{0}\right)^{2}}{v_{F}^{2}}}, m<\Delta\left(\frac{\pi}{d}\right), m \neq$ $0, \Delta\left(k_{z}\right)=\sqrt{\Delta_{S}^{2}+\Delta_{D}^{2}+2 \Delta_{D} \Delta_{S} \cos \left(k_{z} d\right)}$. The form of the spectrum at $v_{0}=0$ is shown in Fig. 33 . Due to the symmetry of the zones, the total chiral magnetic current is zero. In Fig. 3b shows the zero and first LLs in the absence and presence of an electric field. As we can see, the levels become asymmetrical when the electric field is turned on. The degree of such asymmetry of the Landau zero level determines the resulting chiral current. Note that the application of the electric field does not change the distance between the WPs. This means that the relativistic influence of the electric field discussed here has no effect on the anomalous quantum Hall effect. 


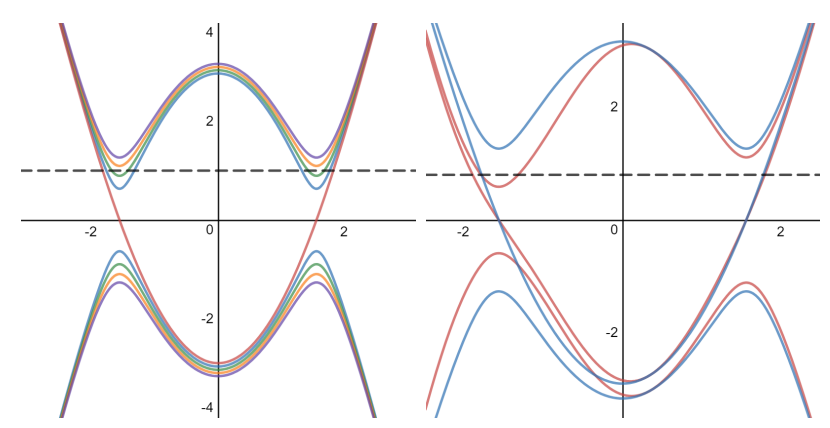

Figure 3. Qualitative picture of LLs in the lattice model. The left side shows LLs in the absence of an electric field along the $\mathrm{Y}$ axis. The right shows LLs in the presence of an electric field. Due to the presence of tilt in the spectrum, the electric field has a different effect on LLs near different WPs. In other words, the symmetric levels become asymmetrical. The degree of such asymmetry of the zero LL determines the magnitude of the resulting chiral current.

As we can see from Fig $3 \mathrm{p}$, the non-zero LLs are still quite symmetrical with respect to the Weyl point for small values of the chemical potential, as well as for small values of $v_{0}$ (we are interested in weak electric field). This means that the contribution from these levels to the total current is negligible. In addition, these levels are not chiral and are sensitive to scattering. Therefore, we consider only the contribution from the zero LLs.

$$
\begin{aligned}
& j_{z}^{0}=\frac{e^{2}}{4 \pi^{2} \hbar^{2}} \frac{B}{c} \int_{-\hbar \pi / d}^{\hbar \pi / d} f v_{z}^{0} d p_{z}= \\
& =\frac{e^{2}}{4 \pi^{2} \hbar^{2}} \frac{B}{c} \frac{1}{\beta} \ln \frac{e^{-\beta\left(\Gamma_{+}-\mu\right)}+1}{e^{-\beta\left(\Gamma_{-}-\mu\right)}+1}
\end{aligned}
$$

where $\Gamma_{ \pm}=\sqrt{1-\frac{\left(\omega_{x} \sin \left(\frac{\pi^{2}}{2 k_{0} d}\right) \mp v_{0}\right)^{2}}{v_{F}^{2}}}\left(m-\Delta\left(\frac{\pi}{d}\right)\right)$. The current vanishes at $v_{0}=0$. In the linear response approximation, we obtain the following expression for the conductivity

$$
\begin{aligned}
& \sigma_{z y}=-\frac{e^{2}}{\pi^{2} \hbar^{2}} \frac{\omega_{x} \sin \left(\frac{\pi^{2}}{2 k_{0} d}\right)}{v_{F}^{2} \Gamma_{0}} \frac{\left(\Delta\left(\frac{\pi}{d}\right)-m\right)^{2}}{e^{\beta\left(\Gamma_{0}-\mu\right)}+1} \\
& \text { where } \Gamma_{0}=\sqrt{1-\frac{\omega_{x}^{2} \sin ^{2}\left(\frac{\pi^{2}}{2 k_{0} d}\right)}{v_{F}^{2}}}\left(m-\Delta\left(\frac{\pi}{d}\right)\right) .
\end{aligned}
$$

\section{CONCLUSION}

Thus, in WSMs with a tilted spectrum apart from the well-known mechanism of transport induction due to the chiral anomaly $(\sim \mathbf{E} \cdot \mathbf{B})$ there is also another mechanism caused by the renormalization of LLs and proportional to the pseudoscalar product of magnetic and electric fields $(\sim \mathbf{E} \vee \mathbf{B})$. At an arbitrary angle between the electric and magnetic fields (when both field-configurations $\mathbf{E} \cdot \mathbf{B}$ and $\mathbf{E} \vee \mathbf{B}$ are non-zero), the total current should consist of two contributions

$$
j_{\mathbf{B}}=\sigma_{R E} \frac{\mathbf{E} \vee \mathbf{B}}{B}+\sigma_{C A E} \frac{\mathbf{E} \cdot \mathbf{B}}{B},
$$

where "B" index means that the electric current is directed along the magnetic field, $\sigma_{R E}$ is the conductivity induced by the previously described effect. " $R E$ " index stands for "Relativistic Effect", because the corresponding part of the electric current results from the relativistic effect of the electric field's impact on LLs. Note that the first term in (32) changes its sign when the vector $\mathbf{E}$ is reflected with respect to the direction of the vector $\mathbf{B}$ $(\alpha \rightarrow 2 \pi-\alpha$, where $\alpha$ is the angle between the $\mathbf{E}$ and $\mathbf{B}$ vectors) and the sign of the second term remains the same. At the same time, when the vector $\mathbf{E}$ is reflected with respect to perpendicular to the magnetic field axis $(\alpha \rightarrow \pi-\alpha)$, the sign of second term changes, and the first remains the same.

Let's make some estimations of the predicted conductivity. We will consider the case with zero temperature. We suppose that $\epsilon_{F}=100 \mathrm{meV}, v_{F}=10^{8} \mathrm{~cm} / \mathrm{sec}$, $\omega_{x}=\omega_{y}=0.5 v_{F}$. We should note that with these parameters the energy of the first Landau level exceeds the Fermi level in fields $B>1 \mathrm{~T}$. In this case we can take into account only the zero LL and use formula (11): $\sigma_{z y} \simeq 10^{13} \mathrm{sec}^{-1}$. Using the formula (13) we can estimate the carrier mobility through the considered transport channel. For the parameter values we use here the estimations provide value $\mu_{z y} \simeq 10^{4} \frac{\mathrm{cm}^{2}}{\mathrm{~V} \cdot \mathrm{sec}}$ at $B=1 \mathrm{~T}$. Note that conductivity $\sigma_{z y}$ decreases with increasing magnetic field intensity. This can be explained that the difference between velocities of zero LLs for different WPs decreases as well.

The effect described above results from chiral zero LLs. This is precisely the reason we called it the chiral current. For example, in a Dirac semimetal this effect should be non-existent.

We should note that such current will not appear with respect to gauge pseudo-fields induced by deformations [27 31]. Indeed, pseudo-fields appear with different signs in different WPs. Then $v_{0}^{+}=-v_{0}^{-}$. In this case LLs for both WPs are renormalized in an identical manner. In addition, the considered effect will be absent in certain special cases of systems with $4 N$ WPs. The following configuration is an example for such case: $\boldsymbol{\omega}_{+}^{(1)}=\boldsymbol{\omega}_{-}^{(1)}=\left(\omega_{x}, \omega_{y}, 0\right), \boldsymbol{\omega}_{+}^{(2)}=\boldsymbol{\omega}_{-}^{(2)}=\left(-\omega_{x},-\omega_{y}, 0\right)$, where superscripts $(1,2)$ denominate the Weyl pair numbers.

Finally, the fact that the proposed transport mechanism is directly related to the tilt in the spectrum gives potential application possibilities. In particular, the study of this transport can become an effective tool for identify and study of the tilt in the spectrum. By changing the directions of the electric and magnetic fields, one can get quite rich information about the tilt parame- 
ters.We should note that considering a stricter theory of the describe effect is of certain interest. The stricter theory should account for tilt along the $Z$ axis as well. The latter may result in a non-zero contribution from non-zero LLs. Moreover, the Shubnikov-de Haas oscillations should be accounted for. All these effect are of paramount interest, but the essence of the effect de- scribed in this work evidently will not change at the qualitative level.

I thank Dmitry Kharzeev and Alexey Soluyanov for the useful discussion of the work. This work was supported by grants RFBR 18-02-01022a, 18-32-00205 mol(a), 1902-01000 A.
[1] C.L. Kane and E.J. Mele, Phys. Rev. Lett. 95, 146802 (2005)

[2] B.A. Bernevig, T.L. Hughes, and S.-C. Zhang, Science 314, 1757 (2006)

[3] L. Fu, C.L. Kane, and E.J. Mele, Phys. Rev. Lett. 98, 106803 (2007)

[4] M.Z. Hasan and C.L. Kane, Rev. Mod. Phys. 82, 3045 (2010)

[5] X.-L. Qi and S.-C. Zhang, Rev. Mod. Phys. 83, 1057 (2011)

[6] D.E. Kharzeev and Ho-Ung Yee, Phys. Rev. B 88, 115119 (2013)

[7] S-C. Zhang, J. Hu, Science 294 (5543), 823-828 (2001)

[8] G. E. Volovik, The Universe in a Helium Droplet (Clarendon Press, Oxford, 2003).

[9] G. E. Volovik, Lect. Notes Phys. 718, 31 (2007)

[10] A. A. Burkov, Annu. Rev. Condens. Matter Phys. 9, 359378 (2017)

[11] N. P. Armitage, E. J. Mele, and Ashvin Vishwanath, Rev. Mod. Phys. 90, 015001 (2018)

[12] M. A. Stephanov and Y. Yin, Phys. Rev. Lett. 109, 162001 (2012)

[13] S. Adler, Phys. Rev. 177, 2496 (1969); J. S. Bell and R. Jackiw, Nuovo Cim. A 60, 47 (1969).

[14] H. B. Nielsen and M. Ninomiya, Phys. Lett. 130B, 390 (1983)

[15] G. E. Volovik, Sov. Phys. JETP 65, 1193 (1987)

[16] E.Kharzeev, Prog. Part. Nucl. Phys. 75, 133-151 (2014)
[17] Burkov, A., J. of Phys.: Cond. Matt. 27(11), 113201 (2015)

[18] Soluyanov, A. A., D. Gresch, Z. Wang, Q. Wu, M. Troyer, X. Dai, and B. A. Bernevig, Nature 527(7579), 495 (2015)

[19] Yu, Z.-M., Yao, Y. \& Yang, S. A. Phys. Rev. Lett. 117, 077202 (2016)

[20] Tchoumakov, S., Civelli, M. \& Goerbig, M. O. Phys. Rev. Lett. 117, 086402 (2016)

[21] Tchoumakov, S., Civelli, M. \& Goerbig, M. O. Phys. Rev. B 95, 125306 (2017)

[22] Z.Z. Alisultanov, JETP Letters 105(7) 442-446 (2017)

[23] Z.Z. Alisultanov, Scientific Reports 8(1), 13707 (2018)

[24] Z.Z. Alisultanov, Annals of Physics 392, 196-205 (2018)

[25] A. A. Burkov and L. Balents, Phys. Rev. Lett. 107, 127205 (2011)

[26] A. A. Zyuzin, Si Wu, and A. A. Burkov, Phys. Rev. B 85, $165110(2012)$

[27] Cortijo, A., Ferreiros, Y., Landsteiner, K. \& Vozmediano, M. A. Phys. Rev. Lett. 115, 177202 (2015)

[28] Pikulin, D., Chen, A. \& Franz, M. Phys. Rev. X 6, 041021 (2016)

[29] Rachel, S., Gothel, I., Arovas, D. P. \& Vojta, M. Phys. Rev. Lett. 117, 266801 (2016)

[30] Arjona, V., Castro, E. V. \& Vozmediano, M. A. Phys. Rev. B 96, 081110 (2017)

[31] Cortijo, A., Kharzeev, D., Landsteiner, K. \& Vozmediano, M. A. H. Phys. Rev. B 94, 241405 (2016) 